\title{
Multiple Description Coding Privacy and Quality Preserving Systems
}

\author{
Dr. Aravinda T V ${ }^{1,}$ Mamatha $\mathrm{O}^{2}$ \\ ${ }^{1}$ Professor, Department of CSE (PG), SJMIT, Chitradurga \\ ${ }^{2} \mathrm{M}$. Tech ( $4^{\text {th }}$ semester), Dept. of CSE, SJMIT, Chitradurga
}

\begin{abstract}
With the popularity of mobile wireless devices equipped with various kinds of sensing abilities, a new service paradigm named participatory sensing has emerged to provide users with brand new life experience. However, the wide application of participatory sensing has its own challenges, among which privacy and multimedia data quality preservations are two critical problems. Unfortunately, none of the existing work has fully solved the problem of privacy and quality preserving participatory sensing with multimedia data. In this paper, in this proposing description, which is the first k-anonymous privacy preserving scheme for participatory sensing with multimedia data? Description integrates a data coding technique and message transfer strategies, to achieve strong protection of participants' privacy, while maintaining high data quality. Specifically, we study two kinds of data transfer strategies, namely transfer on meet up (TMU) and minimal cost transfer (MCT). For MCT, in this paper proposing two different but complimentary algorithms, including an approximation algorithm and a heuristic algorithm, subject to different strengths of the requirement. Furthermore, we have implemented description and evaluated its performance using publicly released taxi traces. The evaluation results show that description achieves high data quality, with low computation and communication overhead.
\end{abstract}

Keywords: Participatory sensing, privacy preservation, K-anonymity, erasure coding

\section{Introduction}

The wide application of mobile communication equipments and the fast advance of sensing technologies have led to the wide availability of privately-held, low cost, advancedprocessing, and big-storage mobile wireless devices, that are equipped with a number of embedded sensors (e.g. microphone, camera, accelerometer, gyroscope, and GPS). On one hand, modern wireless communication technologies (e.g., 2G/3G/4G, Wi-Fi, and Bluetooth) make the communication between mobile devices and infrastructure, as well as between mobile devices themselves, convenient and fast. On the other hand, the mobile devices, especially smart phones, are no longer a tool only for communication, but "computers" with multifunction. Participatory sensing [1] emerged as a new service paradigm using human-carried mobile devices, such as smart phones, for distributed data collection, exchange, analysis, and sharing. With an estimated number of $6: 8$ billion mobile cellular subscriptions worldwide [2], participatory sensing may provide an unprecedented spatial coverage, with very low or even no deployment cost.

On the other hand, the mobile devices, especially smart phones, are no longer a tool only for communication, but "computers" with multifunction. Participatory sensing [1] emerged as a new service paradigm using human-carried mobile devices, such as smart phones, for distributed data collection, exchange, analysis, and sharing. With an estimated number of 6:8 billion mobile-cellular subscriptions worldwide [2], participatory sensing may provide an unprecedented spatial coverage, with very low or even no deployment cost. Compared with traditional decentralized data collection methods (e.g., wireless sensor networks), participatory sensing demonstrates several outstanding advantages, including larger coverage, lower cost, mobile capability, more sufficient energy supply, and more flexible interactive capability. In this paper, here present description, which is a coding, based k-anonymous privacy preserving scheme, working on application layer, for participatory sensing with multimedia data. Intuitively, kanonymity means that the service provider cannot identify the contributor of each sensing record from a group of at least $\mathrm{k}$ participants. Description integrates a data coding technique and message exchanging strategies, to achieve strong protection of participants' privacy, while maintaining high data quality and inducing low communication and computation overhead. In this paper implementing description technique in order to provide privacy for multimedia data and by description achieves high data quality, with low computation and communication overhead.

Compared with traditional decentralized data collection methods (e.g., wireless sensor networks), participatory sensing demonstrates several outstanding advantages, including larger coverage, lower cost, mobile capability, more sufficient energy supply, and more flexible interactive capability. Attracted by the practical and commercial value of participatory sensing, many participatory sensing applications have appeared. For instance, GreenGPS [3] provides the most fuel-efficient routes to drivers; PEIR [4] presents a personal environmental impact report for every individual; PEPSI [5], [6] introduces a privacy enhanced infrastructure for participatory sensing system; ART Sense proposes an anonymous reputation and trust mechanism for participatory sensing; and Ikarus uses sensor data collected during cross-country flights via participatory sensing applications to study thermal effects in the atmosphere, and Pool View gives a privacy preserving architecture for stream data collection. In addition, participatory sensing has been widely used in many practical situations [1], for instance, environment measurement, health care, traffic monitoring, community service, crowd sourcing, and so on. However, the application of participatory sensing has a number of challenges. One of the major challenges is on privacy preservation. Sensing record sent to the service provider, is 


\section{International Journal of Science and Research (IJSR) \\ ISSN (Online): 2319-7064 \\ Index Copernicus Value (2013): 6.14 | Impact Factor (2015): 6.391}

usually attached with spatio-temporal tags indicating the location and time information of the data collected. However, a corrupt service provider may infer private information of the participants, such as identity, home and office addresses, traveling paths, as well as participants' habits and lifestyles, from the sensing records. In turn, many users are reluctant to contribute any sensing record if proper privacy preservation scheme is not applied. Without sufficient number of participants, participatory sensing applications cannot guarantee their quality of services at the expected level.

Therefore, designing privacy preserving schemes for participatory sensing is highly important. Another major challenge is on the variety of sensing data. Most of existing applications of participatory sensing only collect small pieces of sensing data (e.g., temperature, velocity, and geographic location). However, more and more newly emerged applications rely on collecting information of surrounding environment in the format of multimedia (e.g., digital image and video), which result in much higher volume of sensing data. Simply applying existing privacy preserving schemes to participatory sensing with multimedia data is not satisfactory, since existing schemes either induce unacceptable amount of communication cost, or degrade the utility/ quality of the data badly, in case of multimedia sensing.

In this paper, presenting description, which is a coding, based k-anonymous privacy preserving scheme, working on application layer, for participatory sensing with multimedia data. Intuitively, k-anonymity means that the service provider cannot identify the contributor of each sensing record from a group of at least $\mathrm{k}$ participants. description integrates a data coding technique and message exchanging strategies, to achieve strong protection of participants' privacy, while maintaining high data quality and inducing low communication and computation overhead.

- In this paper proposing description for participatory sensing with multimedia data, to achieve both kanonymous privacy preservation and high data quality, with low communication and computation overhead.

- Design an erasure coding based sensing record coding scheme to encode each sensing record into a number of data slices, each of which can be delivered to the service provider through the other participants or the record's generator herself. When a proper data slice exchanging strategy is applied, the contributor of each particular sensing record is hidden in a group of at least $\mathrm{k}$ participants.

- In this paper proposing two kinds of strategies for slice transfer. The first and straightforward strategy is named Transfer on Meet up (TMU), which is to transfer a slice upon meeting another participant. The latter delivers the slice to the service provider.

\section{Related Work}

\section{a) Privacy Preserving Techniques}

In the current state-of-the-art, a number of privacy preserving techniques for participatory sensing systems, especially the location-based services (LBSs), have been proposed by previous researchers, mainly to address the privacy of data source identity, user location, user trajectory, and sensing data content itself. These techniques can be classified into the following four categories.

\section{b) Randomization Based Techniques}

Randomization (noise) based technique where noise (e.g., Gaussian noise) may be added into the original data, can hide the real value of sensitive information (e.g., the trend of the data over time). This method was widely studied and used in data mining field. However, the loss of data quality is a significant shortcoming.

\section{c) Generalization}

The k-anonymity model, which aims to hide each user's sensitive information among k 1 others', is a universal metric for privacy preservation, and has been applied to participatory sensing in several previous work. However, this kind of method usually needs an honest third-party as the anonymizer, which is not allowed in ubiquitous semihonest models. Therefore, when a more severe situation of semi-honest third-party is considered, these approaches cannot meet requirements.

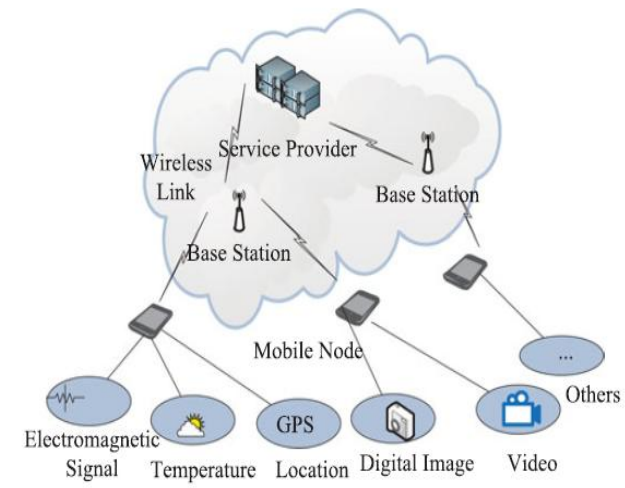

Figure 1.1: System Model: The architecture of cloud-based participatory sensing

Consider a cloud-based participatory sensing and service framework as shown in Figure 1.1 in which there is a service provider and a number of mobile nodes/participants equipped with different kinds of sensors. The service provider aggregates, classifies, analyzes, and stores sensing records reported from the participants, and provides query services based on the records. A mobile node/participant is a user carrying a portable and wireless enabled device (e.g., smart phone, tablet, and laptop). In this project, we use mobile node and participant interchangeably.

\section{Conclusion}

In this paper, we have presented a coding-based privacy preserving scheme, namely description, which is a kanonymous privacy preserving scheme for participatory sensing with multimedia data. description integrates the technique of erasure coding and well designed slice transfer strategies, to achieve strong protection of participant's private information as well as high data quality and loss tolerance, with low computation and communication overhead. here have studied two kinds of data transfer strategies, including TMU and MCT. While TMU is a simple and straightforward strategy, MCT contains two complimentary algorithms, including an approximation

\section{Volume 5 Issue 6, June 2016}




\section{International Journal of Science and Research (IJSR) \\ ISSN (Online): 2319-7064}

Index Copernicus Value (2013): 6.14 | Impact Factor (2015): 6.391

algorithm and a heuristic algorithm, designed for satisfying different levels of delivery guarantee. here also implement description and evaluate its performance using publicly released taxi traces. The evaluation results confirm that description achieves high data quality, strong robustness, with low computation and communication overhead. For future work, one possible direction is to study the problem of privacy preservation in the query process, and design new privacy preserving query schemes based on description. We also think about the lost-packet authentication in server side to increase the construction ratio and further reduce the communication overhead. Another possible direction is to design efficient slice transfer algorithm, considering the limitation of mobile devices. Battery power, storage space, availability, computation ability, and communication bandwidth.

\section{References}

[1] J. Burke, D. Estrin, M. Hansen, A. Parker, N. Ramanathan, S. Reddy, and M. B. Srivastava, "Participatory sensing," presented at the First Workshop World-Sensor-Web 4th ACM Conf. Embedded Netw. Sen. Syst., Boulder, CO, USA, Oct. 2006.

[2] "The world in 2013: ICT Facts and Figures," International Telecommunication Union. [Online]. Available: http://www.itu.int, 2013.

[3] R. K. Ganti, N. Pham, H. Ahmadi, S. Nangia, and T. F. Abdelzaher, "GreenGPS: A participatory sensing fuelefficient maps application," presented at the 8th Int. Conf. Mobile Syst., Appl. Serv., San Francisco, CA, USA, Jun. 2010.

[4] M. Mun, S. Reddy, K. Shilton, N. Yau, J. Burke, D. Estrin, M. Hansen, E. Howard, R. West, and P. Boda, "PEIR: The personal environmental impact report, as a platform for participatory sensing systems research," presented at the 7th Int. Conf. Mobile Syst., Appl., Serv., Krakow, Poland, Jun. 2009.

[5] E. D. Cristofaro and C. Soriente, "Pepsi: Privacy enhancing participatory sensing infrastructure," presented at the 4th ACM Conf. Wireless Netw. Secur., Hamburg, Germany, Jun. 2011.

[6] E. D. Cristofaro and C. Soriente, "Extended capabilities for a privacy-enhanced participatory sensing infrastructure (pepsi)," IEEE Trans. Inf. Forensics Security, vol. 8, no. 12, pp. 2021-2033, Assug. 2013.

Volume 5 Issue 6, June 2016 www.ijsr.net 